\section{Fuzzy computing felt to be the next step in Tokyo}

Tokyo

JAPAN's Ministry of International Trade and Industry (MITI) has persuaded a vast consortium of Japanese companies to invest millions of dollars into establishing a new centre for research and development on fuzzy computing.

The decision to set up the facility, called the Laboratory for International Fuzzy Engineering Research (LIFE), was made at a meeting of MITI officials and industry representatives on 11 January. According to a report in the Nihon Keizai Shimbun (an economic newspaper), 42 companies - including Toshiba, Nippon Steel, Hitachi, and Toyota - will each contribute Y100 million $(\$ 800,000)$ to LIFE. The project, not run directly by MITI, will be chaired in alternating two-year shifts, beginning with Hitachi. The centre, scheduled to open in April in Kannai near Yokohama and expected to operate for six years, will be staffed by some 30 researchers drawn from industry and will conduct joint research with government national laboratories (for example, Tsukuba's Electrotechnical Laboratory) and universities (specifically, Tokyo Institute of Technology and Kyushu Institute of Technology). MITI also plans to collaborate with foreign universities and research asssociations in countries such as France, the United States and West Germany. Patent rights will be held by LIFE and licensed to non-members, but fuzzy algorithms will be made freely available.

Fuzzy computing is based on fuzzy set theory, first presented in 1965 by Professor L.A. Zadeh, a US mathematician at the University of California at Berkeley who is now chairman of the International Fuzzy Systems Association there. Whereas conventional digital computers operate on the binary system, fuzzy computers can form conclusions from imprecise data (such as 'faster' or 'not so hot'), treating such input as having a value somewhere in the continuum between zero and one. Proponents of fuzzy theory hold that these characterisitics will allow fuzzy computers to process information in a manner analogous to the brain.

Considerable research on fuzzy computing has already been carried out in Japan, and fuzzy programming on conventional computers is used to control train speed in a subway in Sendai, a city north of Tokyo. At Kumamoto University, Associate Professor Takeshi Yamakawa has developed a fuzzy microcomputer chip set in collaboration with Omron Tateisi Electronics Company. Of the two chips, the 'rule chip' draws inferences from indistinct data; the 'defuzzifier chip' converts inferred conclusions into analog numerical values. In September 1987, the US National Aeronautics and Space Administration (NASA) asked Yamakawa if it could use his technology in controlling attitude and docking in the space shuttle. Yamakawa is said to have agreed on condition that the chips be used solely in civilian applications. NASA is said to have complied. Sometime this spring - after NASA completes its evaluation - Yamakawa intends to commercialize the device.

"What we are interested in falls into three categories", says MITI spokesman Taizo Nishikawa, deputy director of the Electronics Policy Division at the Machinery and Information Industries Bureau, "fuzzy control, such as the Sendai system, fuzzy information processing; and fabrica-

\section{London}

RoBERTo Poljak of the Pasteur Institute in Paris, Walter Schaffner of the University of Zurich and Gregory Winter of the Medical Research Council's Laboratory of Molecular Biology in Cambridge are to share the 1989 Louis Jeantet Prize in Medicine, awarded for outstanding biomedical research in Western Europe. All three are selected for their contributions to immunology, and each will receive about one-third of the $£ 0.75$ million prize, plus $£ 30,000$ pocket money.

Poljak is cited for his contributions to solving the three-dimensional structure of antibodies, and will use his prize money to improve the computing and graphics power available to his group, and for new area detectors for his X-ray diffraction equipment. He hopes to solve the structure of a lysozyme idiotope-anti-idiotope complex. Schaffner will invest in an automated cell-microinjection apparatus and a fluorescence-activated cell sorter, both to enable his group to study the relevance of DNA methylation to the expression of genes, particularly the antibody genes for tion of fuzzy hardware - for example, fuzzy chips - that will be used to evaluate our basic research in the first two categories."

"There is a very broad range of applications for fuzzy computing", continues Nishikawa. "Industrial applications include image processing, plant management, and robotic control. Social applications include medical diagnosis, weather forecasting and aeronautics control."

The scope of the industries from which member companies come is quite broad, as are their specific interests in fuzzy applications. In addition to the steel, electronics and automotive industries represented by the companies listed earlier, members include computer software houses, chemical companies, electrical appliance and consumer electronics manufacturers, transportation companies, an electric power utility, and - very unusual for a MITI technology project a Tokyo securities firm.

Stuart M. Dambrot \& David Swinbanks

\title{
This year's prize season gets under way
}

which he is cited.

Winter is rewarded for work on engineering antibody molecules, and hopes to finance a project he has been hoping to undertake for some years - to design an antibody molecule to bind specifically to an antigen of known three-dimensional structure. Winter has also won the 1990 Emil von Behring prize given by the Philipps University of Marburg. He wins the $£ 6,000$ prize for his contributions to the molecular biology of the development of human monoclonal antibodies.

The 1989 King Faisal International Prizes, each worth $£ 0.5$ million, have also just been announced. The medicine prize is shared by Robert Edwards of the University of Cambridge and Luigi Mastroianni of the University of Pennsylvania School of Medicine for work that has made human in vitro fertilization possible. Theodor Hänsch, who has recently moved from Stanford University to Munich University, and Ahmed Zewail of California Institute of Technology, share the science prize (this year physics) for their laser studies.

Peter Newmark

Poljak (left), Schaffner and Winter: the immune system pays handsome dividends.

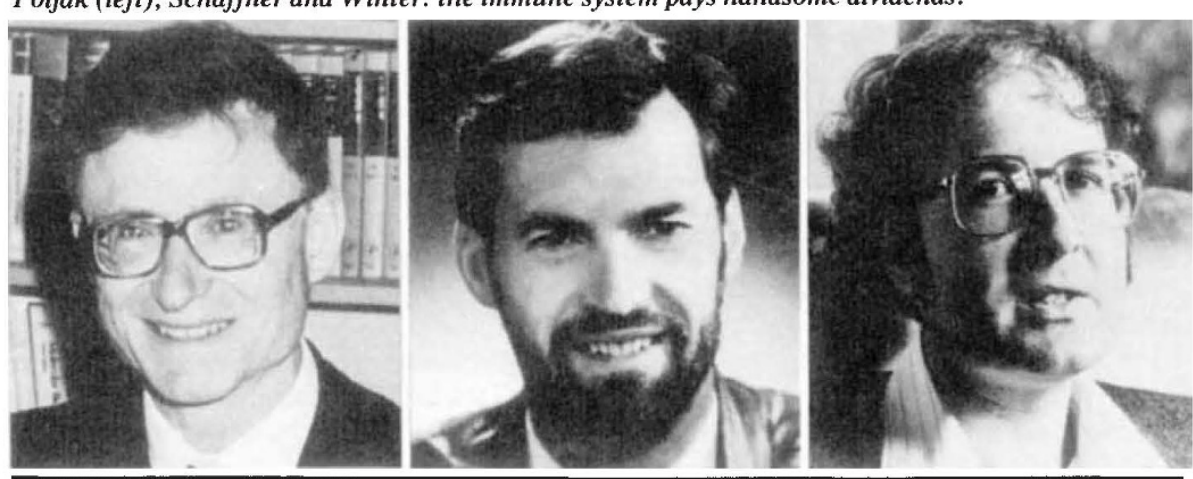

